\title{
Débitmètres ultrasonores pour conduites fermées Evaluations et perspectives d'emploi à EDF
}

\author{
G. Jossinet \\ EDF - Etudes et Recherches - Département REME \\ 25 Allée Privée - Carrefour Pleyel - 93206 Saint Denis
}

\section{Introduction}

Sur les installations de production d'EDF (thermique classique ou nucléaire, hydraulique, etc...) la connaissance des débits de fluide (eau en général) est souvent un paramètre essentiel contribuant directement ou indirectement aux performances et/ou à la sûreté de ces installations. Dans la mesure du possible, les techniques normalisées sont mises en œuvre. Elles sont basées essentiellement sur des méthodes conventionnelles, utilisant des organes déprimogènes : diaphragme, tuyère, venturi.

Ces équipements sont installés à la construction et constituent la majeure partie des équipements de mesure d'exploitation pour assurer les contrôles des process.

Des capteurs d'essais, plus performants sur le plan métrologique, sont associés à ces principes, pour être couramment utilisés dans le cadre des essais de réception des installations (essais contractuels) ou d'essais particuliers de comportement de systèmes élémentaires.

Par ailleurs, une méthode normalisée de mesure de débit par traceur radioactif est utilisée notamment sur les grands diamètres $(0,7 \mathrm{~m}$ à $3 \mathrm{~m})$ où il n'est pas souhaitable ou possible d'installer des organes déprimogènes. Ce procédé est essentiellement utilisé pour la recette d'installation. De par son principe, il est utilisé pour des mesures séquentielles à réaliser en régime stable. Il met en œuvre des moyens conséquents et nécessite des précautions vis-à-vis de l'environnement.

L'intérêt de ces méthodes est de pouvoir garantir une précision de mesure de $\pm 1 \%$ lorsqu'elles sont appliquées suivant leurs normes respectives. Par contre elles font partie des méthodes dites intrusives; leur implantation nécessite des modifications de l'installation telles que la pose de brides, de bossages.

Les débitmètres à ultrasons constituent une voie explorée depuis quelques années, notamment dans la perspective de permettre des mesures de débit de substitution, de complément, ou plus précises sur un grand nombre de conduites non instrumentées à la conception ou encore en conformité imparfaite avec les conditions de la norme NFX 10-102 pour les organes déprimogènes. La débitmétrie par ultrasons peut être considérée comme une méthode non conventionnelle, notamment par le fait que certains procédés sont totalement non intrusifs ou ne nécessitent que peu de modifications des installations.

Au titre des avantages il faut citer:

- l'absence de perte de charges,

- la facilité de mise en œuvre des débitmètres à sondes accrochables (DUSAC),

- le coût pratiquement indépendant du diamètre,

- la possibilité de mesure dans les deux sens de l'écoulement,

- le faible temps de réponse.

Ces techniques sont néanmoins plus complexes. Elles ont atteint une certaine maturité grâce aux progrès de la micro-électronique, à la puissance de calcul et de traitement de signal offerts par la micro-informatique intégrée ainsi que par les avancées technologiques dans le domaine des traducteurs à ultrasons.

L'attrait des éléments précités a conduit EDF/DER à recenser les produits existants, à en sélectionner, à évaluer leurs performances, à engager des développements pour les adapter aux besoins de l'entreprise.

\section{Prospection et domaine d'application à EDF}

Une veille technologique active conduit à suivre l'évolution du marché ; elle a permis de recenser des entreprises et des produits susceptibles d'être utilisés à EDF (tabl. 1). Certains de ces équipements ont fait l'objet d'évaluation en laboratoire sur boucle d'essais et sur site de production nucléaire d'électricité.

\section{Ultrasonic flowmeters for pipes : evaluation and use prospects at EDF}

At EDF/DER, ultrasonic flowmeter with hooking probes (DUSAC in french) have been evaluated on water test loops, at room temperature, at high temperature pressurized water and on nuclear sites.

These instruments may be then used for flow evolution following up.

The multichannel techniques have showed interesting capabilities.

Studies about flow speed profiles are also made. 
Tableau 1.

\begin{tabular}{|c|c|c|c|}
\hline FIRMES & ORIGINE & ÉQUIPEMENTS & OBSERVATIONS \\
\hline $\begin{array}{l}\text { ACCUSONIC } \\
\text { FERRANTI }\end{array}$ & G.B. & 7000 & $\begin{array}{l}\text { - multivoies, très grande conduite } \\
\text { - intrusifs - accrochables en interne }\end{array}$ \\
\hline CALDON & USA & $8300 \mathrm{P}$ & $\begin{array}{l}\text { - multivoies simultanées (2 à } 8 \text { ) } \\
\text { - sondes accrochables } 260^{\circ} \mathrm{C}\end{array}$ \\
\hline CONTROLOTRON & USA & 994 DP & -2 voies, sondes accrochables, $230^{\circ} \mathrm{C}$ \\
\hline DANFOSS & DANEMARK & SONO 2000 & - intrusifs, 2 cordes, $200^{\circ} \mathrm{C}$ \\
\hline FUJI & JAPON & FLB & $\begin{array}{l}\text { - portatif, mono et multivoies (scanner) } \\
\text { - sondes haute température } 230^{\circ} \mathrm{C}\end{array}$ \\
\hline KHRONE & $\begin{array}{l}\text { ALLE- } \\
\text { MAGNE }\end{array}$ & UFM500 & intrusifs, 2 cordes, poste fixe \\
\hline PANAMETRICS & USA & 6468, РТ868 & $\begin{array}{l}\text { - fixe, multivoies (4), séquentiel, intégré } \\
\text { - portatif } 2 \text { voies, }- \text { mesure épaisseur intégrée, } \\
\text { sondes accrochables } 260^{\circ} \mathrm{C}\end{array}$ \\
\hline SEXTANT & FRANCE & DUS $40 / 41$ & - monovoie fixe, bande passante élevée \\
\hline TOKYO KEIKI & JAPON & UFP 1000 , UF 700 & $\begin{array}{l}\text { - monovoie portatif, } 4 \text { voies scanner } \\
\text { - monovoie fixe }\end{array}$ \\
\hline ULTRAFLUX & FRANCE & UF 321 , UF 2100 & $\begin{array}{l}\text { - monovoie, portatif ou fixe } \\
\text { - multivoies (4), séquentiel, intégré } \\
\text { - sondes accrochables }\end{array}$ \\
\hline
\end{tabular}

Les mesures de débits par techniques ultrasonores sont actuellement utilisées, à EDF, pour effectuer des contrôles et des surveillances sur des circuits le plus souvent dépourvus de tout organe de mesure à la construction. Ces mesures sont réalisées par les services techniques de la centrale qui possèdent généralement un ou plusieurs débitmètres de type portable, à sondes accrochables.

L'amélioration de la sûreté des installations conduit à surveiller, en continu, certains circuits dits sensibles, assurant par exemple la réfrigération du cœur à l'arrêt ou la source froide de la tranche. Des projets d'équipement de ces circuits par des débitmètres à sondes intrusives sont en cours.

\section{Quelques considérations métrologiques}

\subsection{Généralités}

Les performances métrologiques d'un débitmètre à ultrasons peuvent être quantifiées à partir des termes : justesse, répétabilité, reproductibilité.

L'incertitude de mesure est obtenue par la somme quadratique de ces trois termes. Le terme reproductibilité est propre à l'utilisation des DUSAC. La reproductibilité permet d'exprimer la dispersion statistique des mesures liée notamment à la mise en place des traducteurs (aspects positionnement et couplage).

\subsection{Paramètres d'influence}

La formulation du débit est rappelée ci-après :

$$
\begin{aligned}
Q=\frac{\Delta T}{\left[T_{a b}-\left(t_{1}+t_{2}\right)\right]\left[T_{b a}-\left(t_{1}+t_{2}\right)\right]} \times & \\
& \times \frac{L}{2 \cos \alpha} \times \frac{\pi \cdot D^{2}}{4} \times K h
\end{aligned}
$$

avec :

- $\Delta T:$ différence de temps de trajet,

- $T_{a b}$ : temps de trajet total sonde amont-sonde aval,

$-T_{b a}:$ temps de trajet total sonde aval-sonde amont,

- $t_{1}:$ temps de trajet dans une sonde,

$-t_{2}:$ temps de trajet dans une paroi,

- $L$ : longueur du trajet dans le fluide,

$-\alpha$ : inclinaison de la ligne de visée avec l'axe d'écoulement,

- $D$ : diamètre interne de la conduite,

- Kh: coefficient de correction hydraulique.

La précision de la mesure dépend donc de trois facteurs :

- les performances de l'électronique $(\Delta T, T)$,

- la définition de l'implantation des sondes et de la section de mesure $(L, \alpha, D)$,

- la connaissance du profil d'écoulement $(K h)$.

\subsection{Bilan prévisionnel d'incertitudes}

L'incertitude sur le débit $\mathrm{d} Q / Q$ peut être exprimée par :

$$
\frac{\mathrm{d} Q}{Q}=\sqrt{\begin{array}{l}
(d \Delta T / \Delta T)^{2}+(2 \mathrm{~d} T / T)^{2}+(\mathrm{d} L / L)^{2}+ \\
(\operatorname{tg} \alpha \cdot \mathrm{d} \alpha)^{2}+(2 \mathrm{~d} D / D)^{2}+(\mathrm{d} K h / K h)^{2}
\end{array} .}
$$


Tableau 2

\begin{tabular}{|l|l|l|l|c|}
\hline \multirow{2}{*}{$\begin{array}{c}\text { ORIGINE DES } \\
\text { INCERTITUDES }\end{array}$} & \multicolumn{2}{c|}{ PARAMĖTRES } & $\begin{array}{c}\text { DUS } \\
\text { Accrochable }\end{array}$ & $\begin{array}{c}\text { DUS } \\
\text { Intrusif }\end{array}$ \\
\hline \multirow{2}{*}{ GÉOMÉTRIE } & diamètre & $\mathrm{d} D / D$ & $0,2 \%$ & $0,2 \%$ \\
\cline { 2 - 5 } & longueur trajet fluide & $\mathrm{d} L / L$ & $0,9 \%$ & $0,05 \%$ \\
\cline { 2 - 5 } & inclinaison & $\operatorname{tg} \alpha \mathrm{d} \alpha$ & $1,5 \%$ & $0,5 \%$ \\
\hline \multirow{2}{*}{ ELECTRONIQUE } & temps trajet fluide & $\mathrm{d} T / T$ & $0,2 \%$ & - \\
\cline { 2 - 5 } & différence temps de trajet & $\mathrm{d} \Delta T / \Delta T$ & $0,5 \%$ & $0,2 \%$ \\
\hline PROFIL ÉCOULEMENT & profil & $\mathrm{d} K h / K h$ & $0,5 \%$ & $0,5 \%$ \\
\hline
\end{tabular}

Un exemple de bilan prévisionnel d'incertitude peut être réalisé pour une application banalisée sans difficultés particulières :

- montages à sondes accrochables et intrusives,

- tuyauterie DN 350 , longueur droite disponible $40 \mathrm{D}$,

- débit $1000 \mathrm{~m}^{3} / \mathrm{h}$.

Dans ces conditions les différents termes d'incertitude sont typiquement voisins des valeurs exprimées dans le tableau 2.

Pour un débitmètre à sondes accrochables dans les conditions précitées, l'erreur de justesse calculée suivant (2) serait de $\pm 2 \%$ de la pleine échelle; elle serait de $\pm 0,8 \%$ pour un débitmètre intrusif (manchette usinée).

\section{Exemples de performances effectives}

Les incertitudes précitées restent toutefois théoriques, et des évaluations expérimentales ont montré la grande sensibilité de ces DUS aux conditions d'écoulement. La détermination des performances effectives passe par une phase d'évaluation expérimentale nécessaire qui conduit à la mise en œuvre de moyens importants.

\subsection{Moyens d'évaluations}

Les différents moyens d'essais employés à EDF/DER pour évaluer divers débitmètres à ultrasons sont les suivants : — une bâche peseuse en eau (DER/CHATOU) :

- capacité $5 \mathrm{~m}^{3}$, débit maximum $200 \mathrm{l} / \mathrm{s}$, précision $\pm 0,2 \%$,

- une boucle d'évaluation «EVEREST » (DER/St DENIS) :

- débit 100 à $1100 \mathrm{~m}^{3} / \mathrm{h}$,

- référence 1 : tuyère à long rayon, précision chaîne de mesure $\pm 1 \%$,

- référence 2 : débit électromagnétique (étalon de transfert), précision $\pm 0,5 \%$,

- pression 4 bar, température $20^{\circ} \mathrm{C}$ à $60^{\circ} \mathrm{C}$.

— une boucle de test de composants «G.B. » (DER/RENARDIÈRES) :

- débit 100 à $600 \mathrm{~m}^{3} / \mathrm{h}$,

- diaphragme, précision de la chaîne de mesure $\pm 1 \%$,
- pression maxi 155 bar, température maxi $295^{\circ} \mathrm{C}$.

Des essais sur site ont été systématiquement effectués avec une mesure de référence, basée sur la méthode du temps de transit de traceurs radioactifs, et mise en cuvre conformément à la norme NF X 10-131.

Dans ces conditions la précision de la mesure de référence est de $\pm 1 \%$.

Tous les essais ont été réalisés à l'aide de systèmes d'acquisition de données permettant une intégration simultanée des mesures de références et des débitmètres sur des périodes de cinq à quinze minutes.

Les DUS ont été évalués dans diverses situations :

- dans des conditions d'implantations favorables : singularité amont à $20 \mathrm{D}$, singularité aval à $10 \mathrm{D}$,

- aux voisinages de singularités,

- sur un circuit en eau pressurisée, à haute température.

\subsection{Evaluations de débitmètres à ultrasons à sondes accro- chables}

Les débitmètres accrochables ont été installés et réglés suivant les recommandations des constructeurs, notamment en ce qui concerne les valeurs du coefficient de correction hydraulique $(K h)$.

\subsubsection{Mesures en conditions de référence}

\subsubsection{En laboratoire}

Les essais sur boucle ont permis d'évaluer les trois termes d'incertitude (justesse, répétabilité, reproductibilité) qui permettent de déduire la précision globale de l'appareil dans les conditions de référence de la norme NFX 10-102 (longueur droite amont $>20 \mathrm{D}$, et aval $>10 \mathrm{D}$ ). De très nombreux essais ont été réalisés sur quatre DUSAC. Un résultat typique est présenté en figure 1 .

Suivant les DUSAC il montre :

- des erreurs de justesse comprises entre $\pm 1 \%$ et $\pm 3 \%$, - des erreurs de répétabilité et reproductibilité confondues situées entre $\pm 1,2 \%$ à $\pm 1,8 \%$,

- l'erreur de répétabilité constatée sur tous les appareils est de l'ordre de $\pm 0,5 \%$.

Ceci correspond à des précisions globales de $\pm 1,6 \%$ à $\pm 3,4 \%$.

\subsubsection{Sur site}

Une intercomparaison entre deux DUSAC et une mesure de débit par traceur ont été menées sur un circuit d'eau 


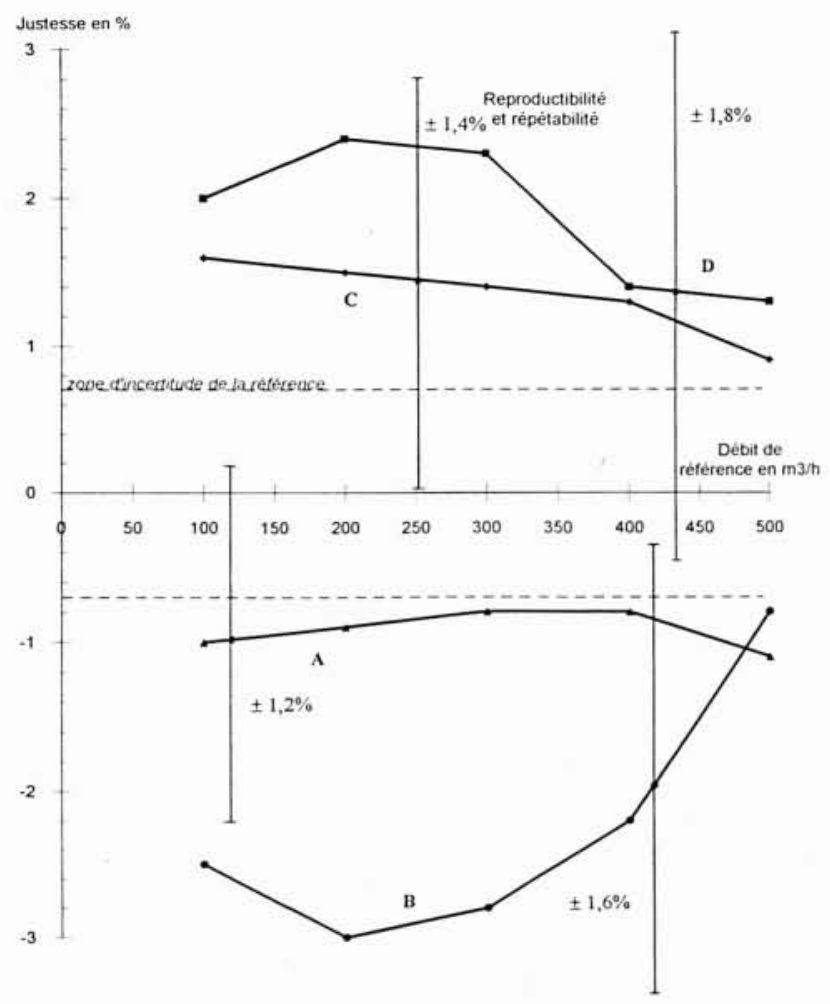

1. Exemples de performances de 4 DUSAC.

brute secouru (SEC) de la centrale de PENLY 1. Ce circuit en eau de mer, classé sûreté, assure par une association d'échangeurs et de deux circuits en cascade (RRI et RRA) l'évacuation de la puissance résiduelle du cœur au cours de l'arrêt de la centrale. L'intercomparaison a été menée au cours des essais de réception du circuit SEC dont les performances thermohydrauliques sont liées à l'encrassement des échangeurs et à la hauteur des marées.

Les caractéristiques essentielles du circuit sont : DN 600, conduite acier à revêtement interne en Néoprène, longueur droite $40 \mathrm{D}$, eau de mer, débit $2000 \mathrm{~m}^{3} / \mathrm{h}$ à $4000 \mathrm{~m}^{3} / \mathrm{h}$. En raison des conditions sévères de propagation acoustique (eau chargée, paroi à revêtement interne, DN 600), les deux DUSAC ont été montés en premier lieu en montage simple trajet, dans deux plans à $45^{\circ}$ par rapport à la verticale. Seul l'un d'eux a pu être disposé en montage reflex grâce à une conception optimale des traducteurs vis-à-vis de l'application.

Les résultats de l'intercomparaison permettent de formuler les remarques suivantes:

- quelles que soient leurs positions, les débitmètres fournissent une mesure par défaut,

- les écarts par rapport à la référence sont de l'ordre de $-3 \%$ à $-6 \%$ pour les deux équipements,

- le montage reflex n'apporte pas d'amélioration significative des performances dans ce cas d'implantation sur $40 \mathrm{D}$ de longueur droite mais il facilite la mise en œuvre, - globalement les équipements sont de classe équivalente; ils permettent d'effectuer, sur une conduite SEC revêtue, une mesure avec une précision de $\pm 6 \%$,

- les performances des deux DUSAC sont moins bonnes sur site qu'en laboratoire $( \pm 3,4 \%)$ : les contrôles dimensionnels et les conditions de pose plus délicats en sont probablement les causes.

\subsubsection{Mesures en présence de singularités}

Les isométries des circuits rencontrés sur sites présentent rarement de grandes longueurs droites. L'utilisateur est donc condamné à disposer le DUSAC aux voisinages de singularités qui modifient profondément la répartition du champ des vitesses à l'emplacement de la mesure.

\subsubsection{Implantation sans précautions particulières}

Au début des années 90 de nombreux tests ont été réalisés dans des conditions de mesures industrielles, en disposant des DUSAC à proximité de diverses singularités (coude, convergent, divergent) disponibles sur la boucle d'essai EVEREST et la bâcle peseuse. Des extraits de ces travaux sont présentés en figure 2 . Ils amènent les remarques suivantes :

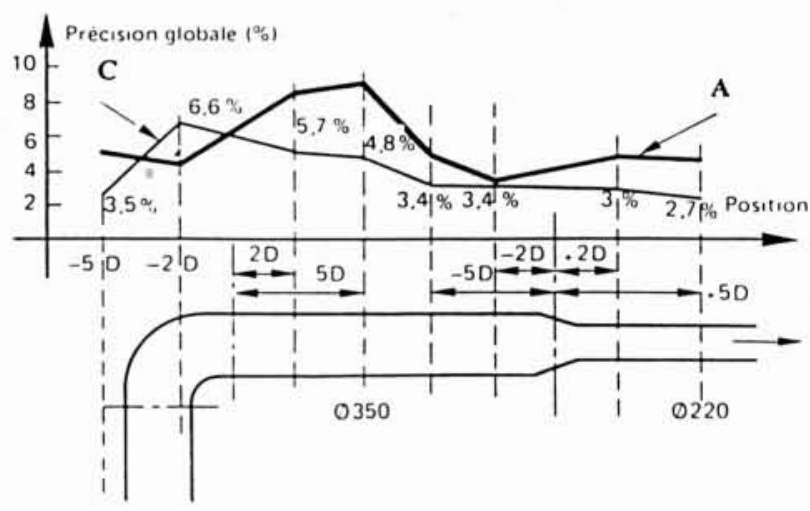

(Essais sur la boucle EVEREST de Saint Denis)

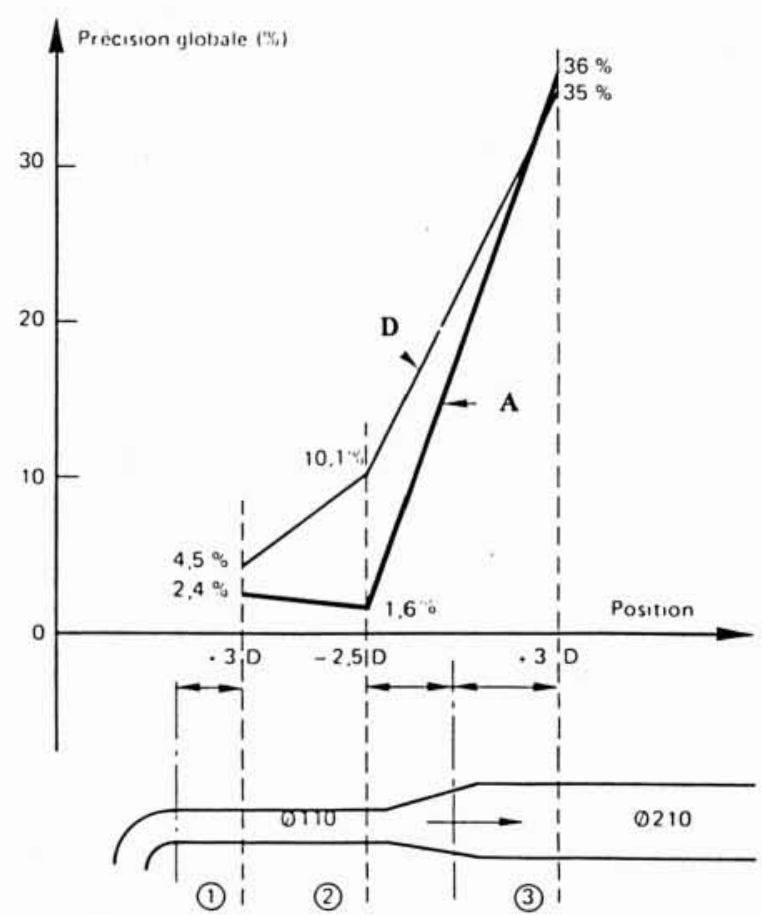

2. Exemples de l'influence des singularités sur la précision globale. 
- les DUSAC placés à des distances inférieures à $5 D$ des singularités ont des précisions globales situées entre $\pm 3 \%$ et $\pm 10 \%$,

- certaines singularités (divergents) conduisent à des erreurs de mesure parfois très importantes telles que $30 \%$.

\subsubsection{Exemples d'amélioration des performances}

Des recherches et des essais plus récents ont montré la possibilité d'améliorer les mesures par la prise en compte de considérations liées à l'influence sur le DUS des composantes de vitesses non-débitantes.

Des mesures systématiques de composantes de vitesses radiales sont effectuées à l'aide du DUSAC et de traducteurs ultrasonores plans disposés sur des lignes de visées diamétrales. Les montages des DUSAC (simple et reflex) sont effectués dans les plans à vitesse radiale maximale et minimale.

La figure 3 illustre une situation de mesure entre deux coudes coplanaires:

- les mesures effectuées dans le plan à vitesse radiale maximale sont entachées d'une erreur importante. Elle est pratiquement éliminée (justesse $<1 \%$ ) par moyennage des mesures croisées simples,

- les résultats des montages reflex pris individuellement ou moyennés n'apportent pas une réduction très importante de l'erreur (justesse $<3 \%$ ),

- l'instrumentation dans le plan à vitesse radiale minimale donne de bons résultats (justesse $<1 \%$ ),

- l'apport des montages simples croisés dans ces cas n'est pas significatif.

Des mesures similaires réalisées à débit beaucoup plus faible ont également confirmé ces résultats.

\subsubsection{Mesures sur fluide en haute température}

Des applications potentielles de contrôle et de surveillance existent également à EDF sur certains circuits des réacteurs à eau pressurisée (REP). L'échelle de température se situe entre $150^{\circ} \mathrm{C}$ et $330^{\circ} \mathrm{C}$ avec une zone préférentielle située autour de $220^{\circ} \mathrm{C}$.

Peu de constructeurs offrent actuellement la possibilité d'effectuer des mesures dans de telles conditions; la technologie des traducteurs et de leur couplage, en paroi externe, à haute température constituent les difficultés majeures. Des essais ont été conduits en 1992 et 1993, en collaboration avec trois constructeurs américains (CALDON, CONTROLOTRON, PANAMETRICS) sur la boucle GB (DER/RENARDIÈRES). Ces essais nous ont conduits par ailleurs à concevoir des supports de sondes permettant une mise en œuvre aisée (pose et dépose des sondes à chaud), et à tester des techniques de couplage. Seuls deux équipements (CONTROLOTRON et PANAMETRICS) ont donné des résultats significatifs, le troisième étant en phase de mise au point. Des résultats d'évaluations ont été obtenus notamment entre $210^{\circ} \mathrm{C}$ et $260^{\circ} \mathrm{C}$, pour des montages de sondes en mode reflex sur deux types de tuyauteries en acier inoxydable ( 8 " et $16^{\prime \prime}$ schedule 160). Il apparaît que les deux débitmètres fournissent des mesures par défaut. Le biais systématique est situé généralement entre - $2 \%$ et $-5 \%$ de la valeur lue. Ces résultats sont en accord avec les performances des DUSAC observées à température ambiante.

\subsubsection{Synthèse de comportement des DUSAC}

L'expérience acquise dans le maniement de ces équipements a été capitalisée sous la forme d'un document interne qui intègre des recommandations d'emploi [1]. Les résultats expérimentaux présentés permettent d'avoir une estimation réaliste de la précision des DUSAC en milieu industriel. Le tableau 3 exprime cette estimation compte tenu de notre expérience sur divers DUSAC. L'évolution des techniques et de la connaissance des écoulements, laisse supposer une amélioration des performances de ces appareils.

\subsection{Evaluations de débitmètres à ultrasons multicordes (DUSMC)}

Parmi les débitmètres à ultrasons multivoies (DUSMV) on distingue d'une part les débitmètres dont les lignes de visée multiples, matérialisées par des sondes intrusives, sont des cordes (DUSMC) et d'autre part les débitmètres dont les lignes de visée multiples, matérialisées par des sondes externes accrochables, sont situées dans des plans diamètraux.

La méthode de mesure de débit par ultrasons est sensible à l'influence du profil des vitesses du fait qu'elle explore une section réduite de l'écoulement. Il est possible de minimiser le biais de représentativité intrinsèque de la méthode en explorant le profil des vitesses sur plusieurs cordes : c'est le principe du DUSMC. Après des études comparatives, par modélisation de DUSMC plusieurs configurations d'implantations de cordes ont été retenues dont l'une a fait l'objet d'essais. Une électronique de débitmètre à ultrasons à quatre voies a été associée à une manchette usinée (DN 350) équipée de deux plans de mesure :

- un plan à quatre cordes $( \pm 0,3 R$ et $\pm 0,75 R)$,

- un plan à trois cordes $( \pm 0,75 R-0 R)$.

Tableau 3

\begin{tabular}{|c|c|c|}
\hline \multicolumn{2}{|c|}{ CONDITIONS D'IMPLANTATION } & \multirow{2}{*}{$\begin{array}{c}\text { PRÉCISION GLOBALE } \\
\pm 2 \% \text { à } \pm 4 \%\end{array}$} \\
\hline De référence & $(20 D-15 D)$ & \\
\hline $\begin{array}{l}\text { Au voisinage de singularités } \\
\text { sans choix de position du plan de mesure }\end{array}$ & $(5 D-3 D)$ & $\pm 3 \%$ à $\pm 10 \%$ \\
\hline $\begin{array}{l}\text { Au voisinage de singularités } \\
\text { choix du plan de mesure et montage doub }\end{array}$ & $\begin{array}{l}(5 D-3 D) \\
\text { roisé « } X "\end{array}$ & $\pm 2 \%$ à $\pm 5 \%$ \\
\hline
\end{tabular}




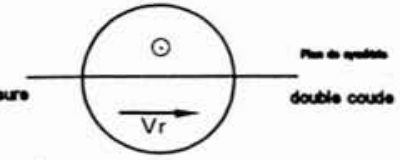

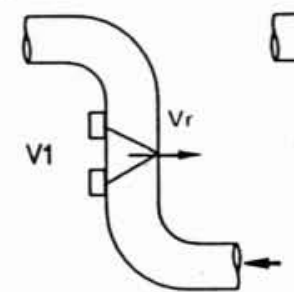

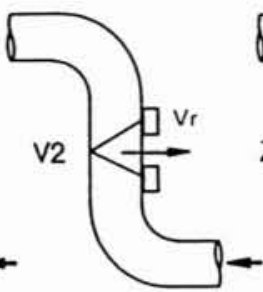

Qm

690

(Cm-Qr)/Qr

(Cm1+Qm2)/2

(Omc-Qr)/Qr

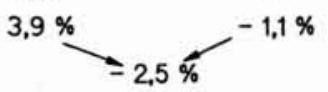

710

$1,1 \%$

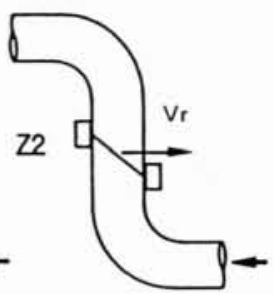

774

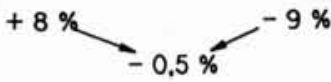

$-0,6 \%$

$-0.5 \%$

2 ZONE A VItEsSE RADiAle MINIMALE

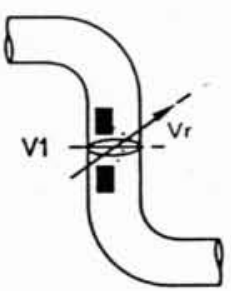

Qm

728

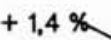

$(\mathrm{Qm}-\mathrm{Qr}) / \mathrm{Qr}$

$(\mathrm{Cm} 1+\mathrm{Qm} 2) / 2$

$(\mathrm{Qmc}-\mathrm{Qr}) / \mathrm{Qr}$

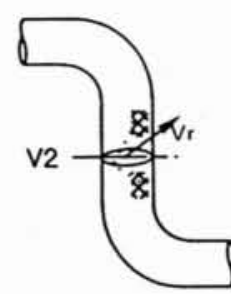

736

$2,5 \%$

3. Exemple d'amélioration de performances.

La détermination des coefficients de pondération $\left(C_{i}\right)$ des vitesses moyennes $\left(V_{i}\right)$ obtenues sur les cordes $\left(l_{i}\right)$ est basée sur une méthode qui suppose :

— un profil de vitesse en écoulement turbulent représenté par:

$$
v=V_{m}(1-r / R)^{p}
$$

avec :

$V_{m}:$ vitesse maximum au centre

$r$ : distance du point courant au centre

$R$ : rayon de la conduite

$p=0,25-0,023 \log \mathrm{Re}$

- une vitesse moyenne sur la section telle que

$V$ section $=\Sigma\left(C_{i} \times V_{i}\right)$;

- un calcul de $V_{i}$ par intégration numérique de (3) le long de la corde de longueur $l_{i}$.

\subsubsection{Mesure en condition de référence}

Les essais ont été réalisés sur la boucle EVEREST ; toutes les singularités (coudes) étant distantes du DUSMC de $\pm 20 \mathrm{D}$. Ils ont permis de comparer, sur une échelle de débit de $75 \mathrm{~m}^{3} / \mathrm{h}$ à $1000 \mathrm{~m}^{3} / \mathrm{h}$, le comportement d'un système configuré en mode monocorde, trois cordes et quatre cordes. Les résultats confirment les performances des DUSMC, comparativement au débitmètre monovoie à corde centrale, sur toute la plage de mesure de 75 à $1000 \mathrm{~m}^{3} / \mathrm{h}\left(70,10^{3}<\operatorname{Re}<10^{6}\right)$. A $10 \%$ de l'échelle de mesure, les écarts par rapport à la référence sont les plus importants :

- monocorde : $+5 \%$,

- trois cordes : $+1,5 \%$,

- quatre cordes : $+0,5 \%$.

\subsubsection{Mesure au voisinage de singularités}

L'évaluation du comportement du même équipement a été réalisée en disposant la manchette de mesure entre deux coudes coplanaires. Des plans de mesure croisés ont été disposés d'une part dans le plan matérialisé par les deux coudes, et d'autre part dans un plan normal. Les résultats font état d'erreurs de mesures comprises entre $\pm 1 \%$ et $\pm 4 \%$ suivant la proximité des singularités. 


\section{DÉBITMÈTRES ULTRASONORES POUR CONDUITES FERMÉES}

\subsubsection{Mesure en température}

Des études et développements ont abouti à la réalisation d'un DUSMC pour circuit en eau pressurisée à haute température. L'originalité réside dans l'interposition de guides d'ondes entre le fluide et les traducteurs à ultrasons. Cette solution présente l'intérêt de repousser largement les limites de fonctionnement en température tout en utilisant des capteurs de technologie courante, travaillant à température ambiante, et de maintenance plus aisée sans arrêt de l'installation. Un tel dispositif à quatre cordes a fait l'objet de tests sur la boucle en eau pressurisée GB (EDF/DER/RNE). Les performances relevées dans des conditions d'installations sévères se traduisent par des écarts de $\pm 1 \%$ à $285^{\circ} \mathrm{C}$. Sur le plan technologique et sur la durée des essais, le comportement des traducteurs s'est avéré sans problème majeur et ceci jusqu'à la limite de fonctionnement en température de la boucle $\left(290^{\circ} \mathrm{C}\right)$. Sur le plan métrologique, les résultats obtenus, compte tenu des singularités peu distantes (divergent à $8 D$ en amont et convergent à $3 \mathrm{D}$ en aval), sont prometteurs.

\subsubsection{Synthèse des essais des DUSMC}

D'un point de vue général, les essais effectués en conditions d'écoulements favorables (singularités de type coude distantes de $\pm 20 \mathrm{D}$ du DUS) confirment les estimations obtenues par simulation.

Sur une dynamique de $100 \mathrm{~m}^{3} / \mathrm{h}$ à $1000 \mathrm{~m}^{3} / \mathrm{h}$, l'erreur de justesse du DUSMC, à manchette usinée en DN 350, reste meilleure que $\pm 1 \%$ de la valeur lue pour un montage à trois cordes. Le DUSMC à quatre cordes reste le plus performant dans les mêmes conditions d'écoulement : sa justesse est voisine de $\pm 0,5 \%$ de la valeur lue. L'amélioration des performances se fait surtout sentir aux faibles débits.

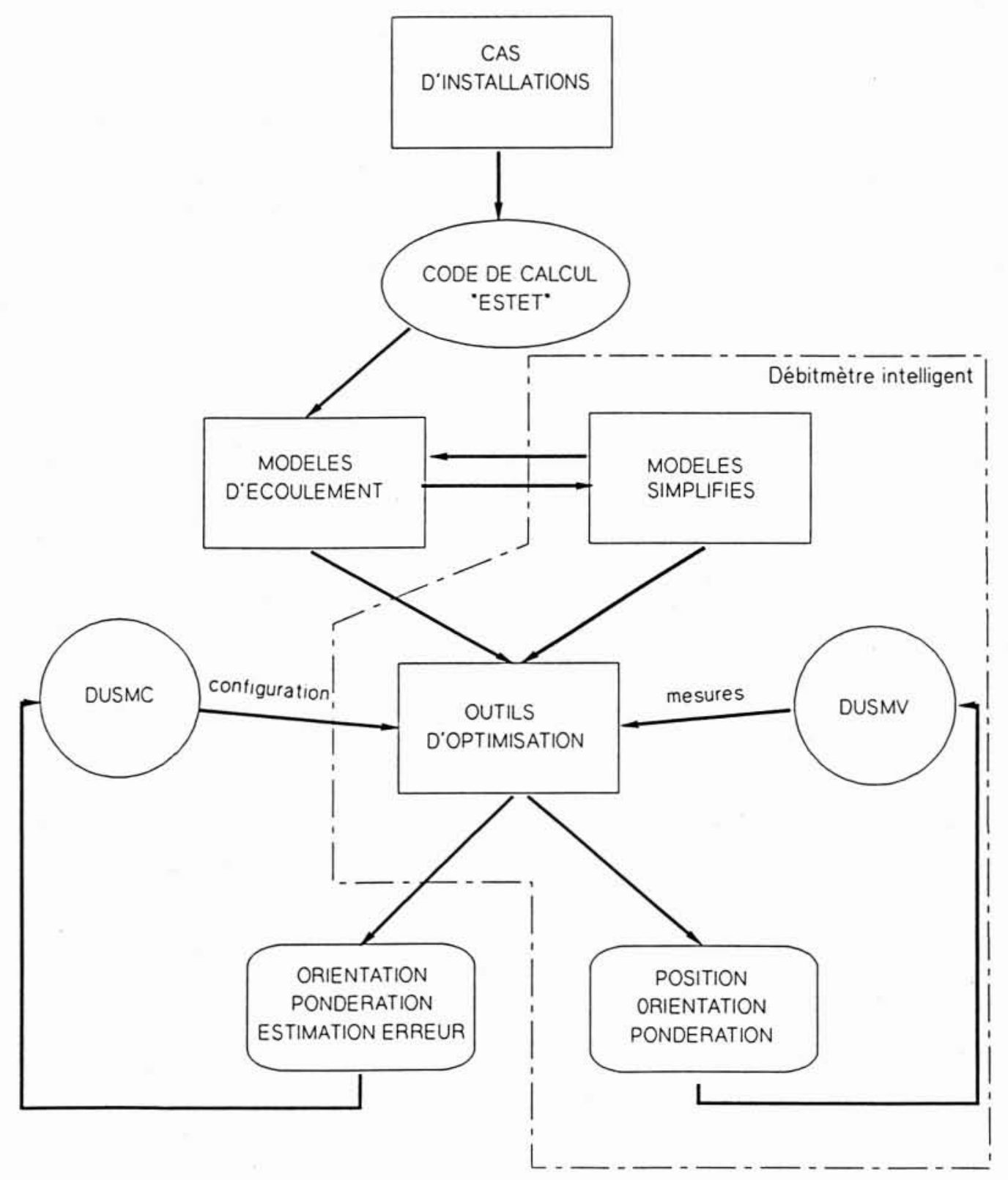

4. Débitmètre à ultrasons - perspectives d'optimisation. 
Les quelques essais réalisés aux voisinages de singularités montrent la nécessité d'utiliser des dispositions de cordes dans des plans croisés pour compenser en partie les composantes de vitesses non-débitantes. Dans le cas de mesures effectuées à proximité et en aval d'un coude, les meilleurs résultats sont obtenus pour des orientations des plans de mesures, normales ou voisines de la normale au plan du coude. Malgré la complexité des systèmes mis en œuvre, les erreurs de mesures constatées dans ces conditions restent encore significatives $( \pm 1 \%$ à $\pm 4 \%)$ au regard des besoins.

\section{Perspectives}

L'analyse des problèmes posés par l'utilisation de la débitmétrie par ultrasons et la volonté d'en améliorer les performances ont amené à effectuer des travaux de recherche sur des méthodes permettant :

- la reconstruction de profils de vitesses d'écoulements à partir de sondages ultrasonores réalisés sur des lignes de visée,

- l'obtention d'un critère de qualité des mesures pour les configurations « conduites-capteurs ",

- l'optimisation de l'instrument considéré comme un ensemble d'éléments (capteurs et lignes de visée) générateurs d'informations [2].

Les études engagées suivent deux démarches synthétisées en figure 4. La première démarche vise à construire un modèle d'écoulement du cas d'installation à l'aide d'un code tridimensionnel d'écoulement («ESTET » EDF/DER/LNH). Une étude paramétrique permet de confronter les configurations du DUS multicordes existant avec le modèle. L'optimisation, qui vise à minimiser l'erreur de mesure, fournit :

- l'orientation des lignes de visées,

- les coefficients de pondération,

- une estimation de l'erreur de mesure.

Une seconde démarche utilisant les travaux précédents devrait permettre de constituer un ensemble de modèles de type «boîte noire ", fonctions paramétriques ou décomposables dans une base ou famille de fonctions. Ces modèles, portables sur micro-ordinateur, sont associés aux mesures fournies par le débitmètre multivoies (DUSMV). Des algorithmes de reconstruction utilisant les mesures permettent de confirmer le modèle retenu et de fournir une configuration plausible et optimale des lignes de visée (position, orientation, pondération). L'ensemble de ces fonctions d'aide à l'utilisateur pourrait être intégré dans un même équipement pour constituer un débitmètre à ultrasons intelligent.

\section{Conclusions}

De nombreux essais d'évaluation ont été réalisés sur des boucles d'essais ou sur sites dans des conditions d'installations variées et sur divers débitmètres à ultrasons.
Les débitmètres à ultrasons à sondes accrochables (DUSAC) constituent une variante intéressante des débitmètres pour liquides par leur aspect totalement non intrusif. La facilité de mise en œuvre leur confere des avantages indéniables pour des applications de contrôle et de surveillance.

Les performances métrologiques effectives de ces équipements exprimées par la précision globale (justesse, répétabilité, reproductibilité) sont comprises entre $\pm 2 \%$ et $\pm 5 \%$ dans des conditions de référence. Toutefois, ces performances peuvent être tenues, voire améliorées, par les recommandations suivantes :

- en éloignant si possible la section de mesure notamment des singularités amont ( 15 à $20 \mathrm{D})$ et aval $(5$ à $10 \mathrm{D})$, - par l'adoption de montages à capteurs multiples $(V, X)$, - par l'identification à l'aide de l'instrument lui-même, de composantes de vitesses non-débitantes, et en disposant le plan de mesure dans le plan à vitesse radiale minimale.

Dans la majorité des applications, la tenue en température est plutôt bornée par la stabilité à long terme des produits de couplage à disposer entre les traducteurs et la tuyauterie. Des mesures à court terme (plusieurs jours) peuvent être réalisées jusqu'à $230^{\circ} \mathrm{C}$.

Les débitmètres à ultrasons multivoies, de préférence à sondes intrusives (DUSMC), constituent une voie d'amélioration sur le plan métrologique. Dans des conditions d'implantations et d'écoulements favorables (singularité amont à $20 D$ et singularité aval à $10 D$ ) les dispositifs à quatre voies permettent d'atteindre des précisions voisines de $\pm 0,5 \%$. Dans le cas des mesures au voisinage de singularités, de type coude notamment, les performances constatées se situent entre $\pm 1 \%$ et $\pm 3 \%$.

Par des études complémentaires, il est probable que l'on puisse encore améliorer les performances des débitmètres à ultrasons, dans les cas d'installations sévères, par une optimisation des méthodes de mesures et de l'instrument lui-même.

Enfin il est à noter que les méthodes de mesures des débits par ultrasons souffrent de l'absence de règles d'installation à caractère quantitatif, les axes de développements présentés pourraient aider à cette normalisation.

\section{Références}

[1] G. JossINET. - Mesure du débit des liquides par débitmètre accrochable à ultrasons. Rapport EDF/DER - HP - 12/91.026.

[2] G. Fleury. - Cadre informatif lacunaire - Optimisation de l'instrument - Débitmétrie ultrasonore par temps de vol. Rapport de thèse - Université de Paris XI Orsay. 